\title{
Representações e ancoragens sociais do novo coronavírus e do tratamento da COVID-19 por brasileiros
}

\section{Representations and social anchorages of the new coronavirus and the COVID-19 treatment by Brazilians}

\author{
Emerson Araújo DO Bú1 1D 0000-0003-3864-3872 \\ Maria Edna Silva de ALEXANDRE² ID 0000-0003-3610-7208 \\ Viviane Alves dos Santos BEZERRA² ID 0000-0001-9178-2957 \\ Roseane Christhina da Nova SÁ-SERAFIM³ (ID) 0000-0001-6751-6421 \\ Maria da Penha de Lima COUTINHO² iD 0000-0003-3961-2402
}

\section{Resumo}

Objetivou-se neste estudo apreender a gênese das representações sociais do novo coronavírus, bem como do tratamento da COVID-19, considerando-se diferentes ancoragens sociais de brasileiros. Contou-se com 595 participantes, predominantemente do sexo feminino $(69,9 \%)$ e da região Nordeste do Brasil (64,9\%). Os dados, coletados através de um questionário online, permitiram análises de Classificações Hierárquicas Descendentes, indicando que a gênese das representações sociais do novo coronavírus é marcada por preocupações relativas à sua disseminação e implicações psicossociais e afetivas. Já o campo representacional do tratamento enfatiza a remissão ou a amenização dos sintomas causados pela COVID-19. As variações nas representações sociais identificadas nesta pesquisa, em função dos diferentes grupos sociais, indicam que futuras intervenções devem considerar as especificidades de cada um deles na disseminação de representações e práticas sociais direcionadas para conter o estado pandêmico.

Palavras-chave: Coronavírus; Psicologia social; Psicologia da saúde; Tratamento.

$\rightarrow \nabla$

1 Universidade de Lisboa, Instituto de Ciências Sociais. Av. Prof. Aníbal Bettencourt 9, 1600-189, Lisboa, Portugal. Correspondência para/Correspondence to: E.A. DO BÚ. E-mail: <dobuemerson@gmail.com>.

2 Universidade Federal da Paraíba, Centro de Ciências Humanas, Letras e Artes, Programa de Pós-Graduação em Psicologia Social. João Pessoa, PB, Brasil.

3 Universidade Federal de Campina Grande, Centro de Ciências Biológicas e da Saúde, Unidade Acadêmica de Psicologia. Campina Grande, PB, Brasil

$\checkmark \nabla \nabla$

Como citar este artigo/How to cite this article

Do Bú, E. A., Alexandre, M. E. S., Bezerra, V. A. S., Sá-Serafin, R. C. N., \& Coutinho, M. P. L. (2020). Representações e ancoragens sociais do novo coronavírus e do tratamento da COVID-19 por brasileiros. Estudos de Psicologia (Campinas), 37, e200073. http:// dx.doi.org/10.1590/1982-0275202037e200073 


\begin{abstract}
This study aimed to apprehend the genesis of the Social Representations of the new coronavirus, as well as of the treatment of the COVID-19, considering Brazilian people's different social anchorages. For that purpose, an online questionnaire was answered by 595 participants, predominantly female (69.9\%) and from the Northeastern region of Brazil (64.9\%). The data collected allowed analyzes of Descending Hierarchical Classifications, indicating that the new coronavirus Social Representations genesis is marked by concerns regarding its dissemination and its psychosocial and affective implications. On the other hand, the representational field of the treatment emphasizes the remission or alleviation of symptoms caused by COVID-19. Given the differences between social groups, the Social Representations variations identified in this research indicate that future interventions should consider each group's specificities in the dissemination of representations and social practices aiming at containing the pandemic state.
\end{abstract}

Keywords: Coronavirus; Treatment; Social psychology; Health psychology.

A Coronavirus Disease 2019 (COVID-19) tem despertado a atenção mundial, ocupando significativo espaço na mídia, na hipermídia e, sobretudo, nas conversações cotidianas de diferentes grupos sociais (Correia, Ramos, \& Bathen, 2020). Trata-se de um problema de saúde coletiva, com sérias implicações para a saúde pública, que tem provocado modificações no estilo de vida da população, principalmente no que tange às interações sociais entre pares, dada a recomendação do distanciamento físico para prevenção e contenção do vírus (Brooks et al., 2020; Duan \& Zhu, 2020; Fiorillo \& Gorwood, 2020).

O referido fenômeno, primeiramente, passou a fazer parte da dinâmica social dos chineses em meados de dezembro de 2019. Todavia, em poucos meses, o Severe Acute Respiratory Syndrome Coronavirus 2 (SARS-CoV-2) se espalhou pelos cinco continentes, levando a Organização Mundial da Sáude a declará-lo como uma emergência de saúde pública internacional, dado o seu estado pandêmico (Velavan \& Maeyer, 2020; Xu et al., 2020; World Human Organization [WHO], 2020).

Em termos operacionais, o SARS-CoV-2 provoca a COVID-19, que consiste em uma doença causada por uma grande família de coronavírus, microrganismo que afeta humanos e atua como agente infeccioso com alto índice de contágio e mortalidade (Velavan \& Maeyer, 2020; WHO, 2020). A sua transmissão ocorre de pessoa para pessoa de forma rápida, e seu controle representa um grande desafio (Xu et al., 2020).

Prevalentemente, as pessoas com diagnóstico de COVID-19 desenvolvem uma síndrome respiratória aguda, classificada em leve, moderada ou grave. Os fatores de risco mais preponderantes para a agudização dos casos são as doenças cardiovasculares, metabólicas, pulmonares, hepáticas e renais (Villegas-Chiroque, 2020). Dados epidemiológicos indicam que $80 \%$ da população infectada apresenta quadros de pneumonia atípica de leve a moderada, 15\% evoluem para uma pneumonia grave e 5\% dos casos podem desenvolver a Severe Acute Respiratory Syndrome (SARS, Síndrome Respiratória Aguda Grave). Na fase crítica da doença, muitos desenvolvem sepse (infecção generalizada no organismo humano), entram em choque e morrem (Velavan \& Maeyer, 2020; Villegas-Chiroque, 2020).

Em relação à sintomatologia, as pessoas infectadas apresentam sintomas respiratórios e gastrointestinais após um período de incubação que varia de cinco a catorze dias (Huang, Wuang, Xingwang, Ren, \& Zao, 2020). Sua clínica inclui, principalmente, febre ao início do quadro infecioso, tosse seca e dispneia (dificuldade para respirar). Adicionalmente, a pessoa contaminada pode queixar-se de mialgia, fadiga, mal-estar e diarreia (Velavan \& Maeyer, 2020). No que tange à suscetibilidade de ocorrência da COVID-19, estudos apontam que homens idosos e imunodeprimidos são os mais suscetíveis (Velavan \& Maeyer, 2020; Villegas-Chiroque, 2020). As crianças, por sua vez, são menos vulneráveis à contaminação pelo vírus. Todavia, crianças e jovens, quando infectados, podem permanecer assintomáticas e funcionarem como agentes transmissores do SARS-

2 CoV-2 para outras pessoas (Li et al., 2020). 
Além das questões epidemiológicas, faz-se importante pontuar também os aspectos relacionados às medidas profiláticas para a prevenção e o controle da velocidade de contágio do novo coronavírus. Nessa direção, as principais recomendações são o distanciamento físico, o confinamento domiciliar, a prática de higiene das mãos, o uso de máscaras e a detecção precoce de pessoas infectadas (Adhikari et al., 2020; Duan \& Zhu, 2020). Quanto ao tratamento para a COVID-19, o que se tem até o momento são planos terapêuticos de suporte para a sintomatologia que ela provoca. Como ainda não se dispõe de um tratamento farmacológico com eficácia terapêutica e evidências científicas comprovadas em larga escala, o que existe são estudos preliminares que apresentam uma possibilidade interventiva. Ademais, não há estudos conclusivos sobre a imunização (Adhikari et al., 2020; Mahase, 2020). Esse panorama desperta preocupação na população mundial, desencadeia ou potencializa desajustes socioafetivos e transtornos psicológicos preexistentes. Assim, as pessoas ficam mais suscetíveis ao medo, a sensações de insegurança e impotência, a quadros de ansiedade, depressão e até tentativas de suicídio (Fiorillo \& Gorwood, 2020; Duan \& Zhu, 2020).

Com base nas elucidações anteriormente descritas, nota-se que o núcleo temático sobre o SARS-CoV-2 e a doença que ele provoca (COVID-19) tornou-se alvo de especulação e estudo das mais diversas áreas do conhecimento, dentre elas a Epidemiologia, a Infectologia, a Saúde Pública e a Psicologia. Portanto, estudar o SARS-CoV-2 e a COVID-19 à luz da Psicologia Social e da Saúde, bem como pensar esses enunciados como signos ou correspondentes simbólicos, inerentes às práticas sociais, permite ao pesquisador apreender o modo como as pessoas se organizam socialmente diante da possibilidade de adoecer e tratar-se (Sá-Serafim, 2013).

Dessa forma, considerando-se a importância do saber do senso comum na compreensão dos temas em saúde (Oliveira, 2000), este artigo busca responder aos seguintes questionamentos: quais são os elementos que compõem a gênese das representações sociais do novo coronavírus e da COVID-19 para brasileiros? Quais são suas compreensões sobre o tratamento de pessoas diagnosticadas com esse vírus? Para responder a tais questões, toma-se como referência a perspectiva psicossociológica, nomeadamente a Teoria das Representações Sociais proposta por Moscovici (2017).

Justifica-se recorrer a esse arcabouço teórico no presente estudo porque se entende que as Representações Sociais são consideradas como princípios que organizam as práticas sociais e as relações simbólicas entre as pessoas frente a objetos sociais que as perpassam (Doise, 2001; Moscovici, 2017). Sabe-se que a gênese de uma representação social se dá por meio de dois processos formadores de natureza social e cognitiva: a ancoragem e a objetivação. Na ancoragem, o indivíduo, em face de um objeto desconhecido, busca em sua memória conteúdos, eventos e pessoas que conhece e os transforma enquanto protótipos, comparando-os com o novo que se interpela. Assim, na ancoragem, assimila-se o novo ao que já existe. Por sua vez, no processo de objetivação, reproduz-se um conceito desconhecido/abstrato da realidade, transferindo-o para um patamar concreto, visível, tangível e "palpável". Nesses dois processos, então, transforma-se o não familiar em familiar (Moscovici, 2017).

No cenário de formação das representações sociais, a mídia apresenta fundamental importância, uma vez que transmite códigos normativos de comunicação e conduta (Moscocivi, 2017). Acerca dessas questões, ressalta-se que, cotidianamente, tem-se verificado, nos mais variados meios de comunicação e interações entre pares, a veiculação e as trocas de informações formais e informais acerca do SARS-CoV-2, bem como da COVID-19. Nesse sentido, sugere-se que tais informações podem ter participação na forma como os brasileiros têm criado e compartilhado representações acerca dos objetos sociais mencionados.

Em face do exposto, o presente estudo direciona esforços para compreender como brasileiros se apropriaram do conhecimento sobre o SARS-CoV-2, bem como do tratamento, ainda que especulativo, da COVID-19, assim que ela começou a fazer parte efetiva de sua dinâmica social, isto é, logo nas três primeiras semanas em que foram testados e confirmados casos com tal diagnóstico no Brasil. Desse modo, compreendendo-se que o campo representacional de um dado objeto/fenômeno é construído a partir das 
interações das diferentes ancoragens sociais da população com ele (Doise, 2001), objetiva-se, no estudo descrito a seguir, apreender as representações sociais dos objetos supramencionados, a partir de diferentes ancoragens sociais de brasileiros (variáveis sociodemográficas). Acredita-se que tais ancoragens atuarão como ideias de força na construção do pensamento social sobre os fenômenos em questão.

\section{Método}

Trata-se de um estudo misto, quantitativo e qualitativo, descritivo e exploratório, ancorado no aporte teórico da Teoria das Representações Sociais (Doise, 2001; Moscovici, 2017). A amostra da pesquisa foi composta de maneira não probabilística, por conveniência, mediante a participação de 595 brasileiros, na faixa etária de 18 a 78 anos $(M=29,30 ; D P=10,10)$, predominantemente do sexo feminino $(69,9 \%)$, residentes na região Nordeste do Brasil (64,9\%). Em relação ao grau de escolaridade e à renda, 48,9\% dos participantes possuíam curso superior e 30,1\% auferiam renda de até dois salários mínimos.

Quanto aos instrumentos, utilizou-se um Questionário Sociodemográfico com questões relacionadas a idade, sexo, grau de escolaridade, renda e concentração por região do país; e outro questionário que apresentou a Técnica de Associação Livre de Palavras (TALP), contendo os seguintes estímulos indutores: coronavírus e tratamento de pessoas com coronavírus. Faz-se importante pontuar que a TALP consiste em uma técnica projetiva, que se organiza sobre a evocação de respostas dos participantes, a partir de estímulos indutores previamente definidos pelo pesquisador, possibilitando, assim, identificar universos semânticos relacionados a um objeto ou fenômeno social (Coutinho \& Do Bú, 2017).

Para a coleta de dados, gerou-se um formulário online com os instrumentos supramencionados, divulgado por meio das redes sociais Facebook, WhatsApp e Instagram. Os participantes, após concordarem que eram maiores de 18 anos, brasileiros e que se apresentavam disponíveis para participar da pesquisa voluntariamente, responderam aos dois questionários. Estes ficaram disponíveis para respostas durante cinco dias, entre 14 e 19 de março de 2020, sendo que no dia 14 havia 98 casos da COVID-19 confirmados no Brasil e, no dia 19 de março, 621 casos confirmados e seis mortes no país.

Os dados sociodemográficos foram processados por meio do software IBM ${ }^{\circledR S P S S} \circledast$ Statistics (versão 26), que permitiu realizar análises descritivas. Já para os dados da TALP, utilizou-se o software Interface de $R$ pour les Analyses Multidimensionnelles de Textes et de Questionnaires, que viabilizou o desenvolvimento da análise de Classificação Hierárquica Descendente (CHD).

Destaca-se que a CHD, a partir da análise da relação (testes de $\chi^{2}$ ) entre as palavras evocadas pelos participantes do presente estudo, possibilitou a construção de eixos de significados acerca do novo coronavírus, por meio de classes inter-relacionadas de vocábulos, que se configuram como substância bruta para a análise qualitativa da presente pesquisa. Além disso, essa análise propiciou a observação da construção de cada classe, por meio das relações entre as variáveis de ancoragem (definidas aqui como as características sociodemográficas dos participantes) e as suas evocações (Camargo \& Justo, 2018).

Por fim, fez-se uma análise do conteúdo emergido em cada eixo e classes de palavras oriundas das CHD. Buscou-se, nessa análise qualitativa dos dados, apontar aspectos etimológicos das palavras evocadas e relacioná-los com o que a literatura sobre o tema constata, bem como consideraram-se aspectos contextuais do Brasil na atualidade, de modo a evidenciar e compreender sentidos e significados que são criados e compartilhados pelos participantes do presente estudo em face do novo coronavírus e do tratamento, ainda que especulativo, da COVID-19.

Os procedimentos de coleta de dados seguiram todas as recomendações éticas para esse tipo de pesquisa (CAAE: 30616720.9.0000.0008), conforme preza a Resolução n 510/2016 do Conselho Nacional 4 de Saúde Brasileiro (Ministério da Saúde, 2016). 


\section{Resultados}

Os resultados referentes aos campos representacionais do novo coronavírus e do tratamento da COVID-19 serão apresentados a partir da formação dos eixos temáticos da CHD e de suas respectivas classes, destacando-se também as variáveis de ancoragem social significativas para a formação destas.

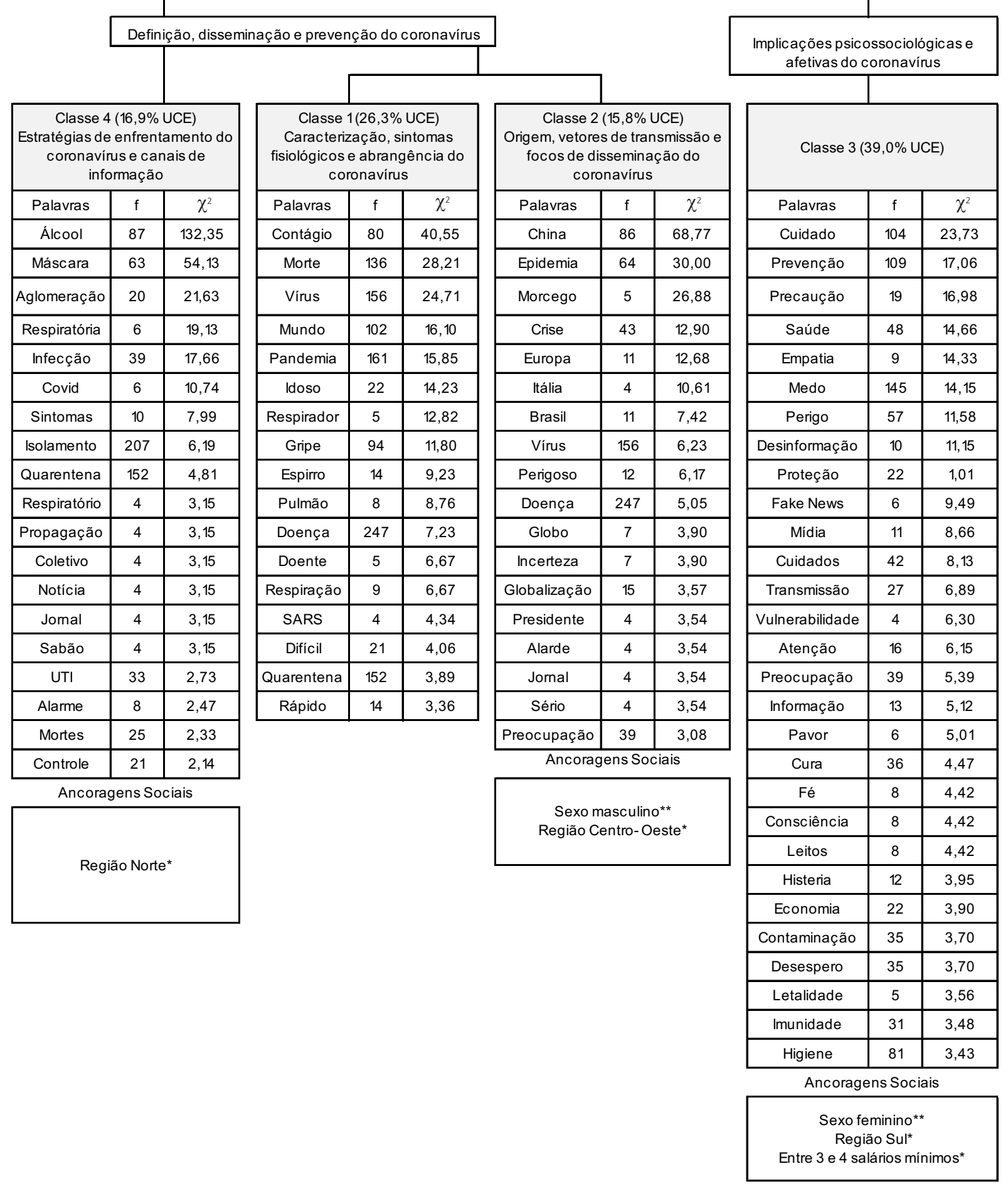

Figura 1. Campo representacional e ancoragens sociais do novo coronavírus. Brasil, 2020.

Nota: ${ }^{*} p \leq 0,05 ;{ }^{* *} p \leq 0,001$. UCE: Unidades de Contextos Elementar. 


\section{Campo representacional e ancoragens sociais do novo coronavírus (SARS-CoV-2)}

No que se refere às evocações dos participantes do presente estudo em face do estímulo "coronavírus", a CHD reteve $79,6 \%$ das Unidades de Contexto Elementar (UCE) do corpus e permitiu identificar quatro classes distintas que compõem o campo representacional do objeto social em questão (Figura 1). Essas classes se apresentam em dois diferentes eixos. O primeiro eixo, intitulado "Definição, disseminação e prevenção do coronavírus", subdivide-se em dois subconjuntos, em que, à direita, encontram-se as classes 1 e 2 e, em oposição, a classe 4. O segundo eixo é composto apenas pela classe 3 e diz respeito às "Implicações psicossociológicas e afetivas do coronavírus" para brasileiros.

A segunda classe, intitulada "Origem, vetores de transmissão e focos de disseminação do coronavírus", concentra 15,80\% das UCE e aborda (majoritariamente a partir de participantes de sexo masculino e da região Centro-Oeste do Brasil as redes de sentido construídas acerca do objeto social em questão. Observa-se nesta classe um intervalo de radicais e vocábulos entre $\chi^{2}=68,77$ (China) e $\chi^{2}=3,08$ (preocupação). A primeira classe (Caracterização, sintomas fisiológicos e abrangência do coronavírus), com 28,27\% das UCEs do corpus, não associou-se a nenhuma das "variáveis de ancoragem" (características sociodemográficas) a priori estabelecidas. Nesse sentido, apresenta objetivações que são consensuais ao grupo de atores sociais deste estudo acerca da COVID-19. Nesta classe, verificaram-se radicais e palavras no intervalo de $\chi^{2}=40,55$ (contágio) a $\chi^{2}=3,36$ (rápido).

Em oposição às classes 1 e 2, mas ainda no eixo "Definição, disseminação e prevenção do coronavírus", encontra-se a classe 4 (Estratégias de enfrentamento do coronavírus e canais de informação), com 16,9\% de retenção de UCE do corpus e intervalo de radicais/palavras de $\chi^{2}=132,35$ (álcool) a $\chi^{2}=3,13$ (sabão). Essa classe apresenta-se como característica das evocações dos participantes residentes no Norte do Brasil.

No eixo 2, evidencia-se a classe 3, que versa acerca das "Implicações sociais, psicológicas e afetivas do coronavírus" para a população brasileira, estando associada, majoritariamente, aos participantes do sexo feminino, com renda entre três e quatro salários mínimos e moradores da região Sul do Brasil. Obteve-se nesta classe o intervalo de radicais e evocações de $\chi^{2}=23,73$ (cuidado) a $\chi^{2}=3,43$ (higiene).

Além de apreender como os participantes deste estudo representam o novo fenômeno que os interpela (SARS-CoV-2), buscou-se também identificar suas representações em relação ao tratamento de pessoas com a COVID-19, considerando, ainda, as diferenças em função das variáveis de ancoragem. Destarte, a seguir, apresenta-se a análise relativa ao estímulo "tratamento de pessoas com coronavírus".

\section{Campo representacional e ancoragens sociais do tratamento de pessoas com coranírus (COVID-19)}

O material coletado, processado a partir da análise de CHD, deu origem ao dendrograma exposto na Figura 2. Tal análise considerou $87,2 \%$ do total das UCE e originou dois eixos. O primeiro eixo, designado "Definições e desafios socioeconômicos face ao tratamento de COVID-19", subdividiu-se e aglutinando as classes 1 (Estratégias de contenção e automedicação), e 2 (Implicações psicossociais e econômicas para o tratamento), em oposição à classe 3 (Itens de proteção, suporte pessoal e assistência médico-hospitalar); enquanto que o segundo eixo "Busca da cura do COVID-19: Instituições e Agentes responsáveis", à esquerda, gerou a Classe 4.

A respeito do eixo 1, especificamente quanto às "Estratégias de contenção e automedicação", verifica-se o intervalo de radicais e evocações entre $\chi^{2}=147,23$ (repouso) e $\chi^{2}=3,07$ (medicação). A característica sociodemográfica dos brasileiros que mais contribuiu para essa classe foi a renda, entre um e dois salários mínimos. No que tange às 'Implicações psicossociais e econômicas para o tratamento", evidenciadas pela 


\begin{tabular}{|c|c|c|}
\hline \multicolumn{3}{|c|}{$\begin{array}{l}\text { Busca da cura da COVID- 19: } \\
\text { instituições e agentes responsáveis }\end{array}$} \\
\hline \multicolumn{3}{|c|}{ Classe 4 (21,7\% UCE) } \\
\hline Palavras & $f$ & $\chi^{2}$ \\
\hline Esperança & 20 & 45,60 \\
\hline Cura & 43 & 37,72 \\
\hline Hospitais & 18 & 31,92 \\
\hline Pesquisa & 27 & 31,05 \\
\hline Medicina & 6 & 20,48 \\
\hline Enfermeiros & 6 & 20,48 \\
\hline Saúde & 62 & 17,18 \\
\hline Governo & 18 & 15,48 \\
\hline Alívio & 4 & 13,60 \\
\hline Sintomas & 10 & 12,85 \\
\hline Urgência & 8 & 12,52 \\
\hline Investimento & 8 & 12,52 \\
\hline SUS & 108 & 11,87 \\
\hline Morte & 136 & 11,10 \\
\hline Idosos & 48 & 10,64 \\
\hline Fé & 11 & 10,60 \\
\hline Ciência & 14 & 9,59 \\
\hline Solução & 7 & 9,49 \\
\hline Socorro & 5 & 9,34 \\
\hline Solidariedade & 5 & 9,34 \\
\hline Importante & 10 & 7,97 \\
\hline Deus & 10 & 7,97 \\
\hline Médicos & 10 & 7,97 \\
\hline Desinformação & 6 & 6,60 \\
\hline Dúvida & 4 & 6,21 \\
\hline Infecção & 32 & 6,11 \\
\hline Dificuldade & 7 & 4,73 \\
\hline Vacina & 98 & 4,15 \\
\hline Vida & 5 & 3,95 \\
\hline Grupo & 5 & 3,95 \\
\hline Conscientização & 8 & 3.39 \\
\hline Doente & 8 & 3,39 \\
\hline \multicolumn{3}{|c|}{ Ancoragens Sociais } \\
\hline \multicolumn{3}{|c|}{ Região Sudeste* } \\
\hline
\end{tabular}

Definições e desafios socieconômicos face ao tratamento da COVID- 19

\begin{tabular}{|c|c|c|c|c|c|}
\hline \multicolumn{3}{|c|}{$\begin{array}{c}\text { Classe } 3 \text { (22,5\% UCE) } \\
\text { Itens de proteção, suporte pessoal e } \\
\text { assistência médico-hospitalar }\end{array}$} & \multicolumn{3}{|c|}{$\begin{array}{c}\text { Classe } 1(34,8 \% \text { UCE) } \\
\text { Estratégias de contenção e } \\
\text { automedicação }\end{array}$} \\
\hline Palavras & $f$ & $\chi^{2}$ & Palavras & $f$ & $\chi^{2}$ \\
\hline Hospital & 104 & 77,83 & Repouso & 91 & 147,23 \\
\hline Máscara & 73 & 35,83 & Hidratação & 21 & 46,69 \\
\hline Álcool & 99 & 27,62 & Água & 18 & 43,94 \\
\hline Quarentena & 167 & 24,65 & Alimentação & 27 & 40,12 \\
\hline Médico & 19 & 20,52 & Descanso & 18 & 36,77 \\
\hline Remédio & 29 & 19,93 & Isolamento & 233 & 32,32 \\
\hline China & 76 & 16,19 & Internação & 16 & 30,32 \\
\hline Lavar & 23 & 16,11 & Paracetamol & 6 & 19,57 \\
\hline Vacina & 98 & 10,04 & Dipirona & 4 & 12,99 \\
\hline Sabão & 7 & 9,63 & Antitérmico & 8 & 11,85 \\
\hline Casa & 23 & 9,58 & Remédios & 21 & 9,99 \\
\hline Gravidade & 9 & 9,06 & Pulmão & 10 & 7,45 \\
\hline Leito & 4 & 8,74 & Imunidade & 30 & 6,82 \\
\hline Sistema & 4 & 8,74 & Paciência & 8 & 6,78 \\
\hline Oxigênio & 4 & 8,74 & Nebulização & 4 & 5,88 \\
\hline Teste & 4 & 8,74 & Chá & 4 & 5,88 \\
\hline Isolado & 4 & 8,74 & Líquido & 4 & 5,88 \\
\hline UTI & 30 & 7,00 & Contágio & 79 & 5,73 \\
\hline Exame & 10 & 6,97 & Consciência & 9 & 5,15 \\
\hline Medicamento & 10 & 6,97 & Tosse & 33 & 4,83 \\
\hline Itália & 20 & 5,29 & Controle & 27 & 4,60 \\
\hline Perigoso & 20 & 5,29 & Gripe & 90 & 4,44 \\
\hline Vitamina & 16 & 4,66 & Vitamina & 16 & 3,68 \\
\hline Brasil & 14 & 4,36 & Desespero & 32 & 3,61 \\
\hline Mídia & 12 & 4,07 & Calma & 8 & 3,12 \\
\hline Cama & 4 & 3,52 & Todos & 8 & 3,12 \\
\hline \multicolumn{3}{|c|}{ Ancoragens Sociais } & Medicação & 20 & 3,07 \\
\hline \multicolumn{3}{|c|}{$\begin{array}{c}\text { Acima de } 8 \text { salários mínimos* } \\
\text { Ensino Superior }\end{array}$} & \multicolumn{3}{|c|}{ Ente 1e 2 salários mínimos* } \\
\hline
\end{tabular}

\begin{tabular}{|c|c|c|}
\hline \multicolumn{3}{|c|}{$\begin{array}{c}\text { Classe } 2 \text { (20,8\% UCE) } \\
\text { Implicações psicossociais e } \\
\text { econômicas para o tratamento }\end{array}$} \\
\hline Palavras & $f$ & $\chi^{2}$ \\
\hline Higiene & 95 & 28,21 \\
\hline Responsabilidade & 25 & 27,37 \\
\hline Cuidados & 44 & 26,15 \\
\hline Medicamentos & 17 & 18,92 \\
\hline Higienização & 21 & 15,98 \\
\hline Medicação & 20 & 9,66 \\
\hline Reclusão & 12 & 9,58 \\
\hline Atenção & 16 & 7,66 \\
\hline Proteção & 25 & 7,55 \\
\hline Limpeza & 22 & 7,50 \\
\hline Evitar & 4 & 6,67 \\
\hline Pobres & 4 & 6,67 \\
\hline Confinamento & 4 & 6,67 \\
\hline Risco & 43 & 6,49 \\
\hline Cuidado & 108 & 6,11 \\
\hline Contato & 18 & 5,58 \\
\hline Tristeza & 10 & 4,73 \\
\hline Colapso & 5 & 4,30 \\
\hline Respeito & 5 & 4,30 \\
\hline Coletividade & 5 & 4,30 \\
\hline Prevenção & 115 & 4,10 \\
\hline Calma & 8 & 3,77 \\
\hline Precaução & 21 & 3,38 \\
\hline Isolamento & 233 & 3,09 \\
\hline \multicolumn{3}{|c|}{ Ancoragens Sociais } \\
\hline \multicolumn{3}{|c|}{$\begin{array}{l}\text { Até um salário mínimo* } \\
\text { Ensino médio* }\end{array}$} \\
\hline
\end{tabular}

Figura 2. Campo representacional e ancoragens sociais do tratamento de pessoas com coronavírus (COVID-19). Brasil, 2020. Nota: * $p \leq 0,05$. UCE: Unidades de Contextos Elementar.

classe 2 da CHD, representativa junto a participantes com Ensino Médio, assim como com renda de até um salário mínimo, destacam-se os radicais e extratos de discurso que objetivam o tratamento no intervalo de $\chi^{2}=28,21$ (higiene) a $\chi^{2}=3,09$ (isolamento).

Em oposição às classes 1 e 2, no eixo 1, encontra-se a classe 3 (Itens de proteção, suporte pessoal e assistência médico-hospitalar) com intervalo de radiais e evocações que variou de $\chi^{2}=77,83$ (hospital) a 
$\chi^{2}=3,52$ (cama). Tal classe desvela evocações dos participantes considerados de maior poder monetário para o contexto brasileiro, com renda per capita superior a oito salários mínimos e Ensino Superior completo. No segundo e último eixo da CHD em análise, encontra-se a classe 4 (Busca da cura da COVID-19: instituições e agentes responsáveis), este eixo fora constituído, majoritariamente, por pessoas que são da região Sudeste do Brasil, com intervalo entre radicais e extratos textuais de $\chi^{2}=45,60$ (esperança) a $\chi^{2}=3,39$ (doente).

\section{Discussão}

As análises das Classificações Hierárquicas Descendentes para os dois estímulos indutores utilizados neste estudo (coronavírus e tratamento de pessoas com coronavírus) permitiram identificar a gênese constitutiva de seus respectivos campos representacionais. Ademais, também foi verificada a suposição de que as variáveis sociodemográficas se configuravam como ideias de força para a construção de contextos representacionais específicos. Desse modo, a partir dos resultados apresentados, é possível reflexionar sobre os campos representacionais dos referidos objetos sociais e, ainda, observar as diferenciações na forma de representá-los em função das variáveis de ancoragem dos participantes.

Especificamente no que se refere aos resultados elucidados por meio da CHD do estímulo indutor coronavírus, observou-se que a classe 1 (Caracterização, sintomas fisiológicos e abrangência do novo coronavírus) apresenta o que foi mais consensual na representação do objeto entre os diferentes grupos sociais desta pesquisa. Nesse sentido, definiu-se o SARS-CoV-2, no momento de coleta dos dados, como um vírus de rápido alastramento pelo mundo (pandemia), com sintomas e forma de contágio característicos de uma gripe (espirro). Tal vírus parece estar associado àquele que provoca uma doença nos pulmões, que pode evoluir, principalmente em idosos, para um quadro de dificuldade respiratória, necessitar do auxílio de respiradores para o seu tratamento e levar à morte. Destarte, demonstra-se uma apropriação do que tem sido veiculado na literatura, principalmente biomédica, acerca do SARS-CoV-2 e do que ele provoca em pessoas contaminadas (Huang et al., 2020; Villegas-Chiroque, 2020).

A segunda classe (Origem, vetores de transmissão e focos de disseminação do coronavírus), ainda que contida no mesmo subconjunto que a classe 1, apresenta especificidades dos participantes do sexo masculino e daqueles residentes no Centro-Oeste do Brasil. Para esses grupos, o vírus SARS-CoV-2 surge na China como uma epidemia e, dada a globalização, dissemina-se para a Europa, com especial foco na Itália, chegando, finalmente, ao Brasil. O novo vírus interpela-se para esses grupos como uma incerteza, sendo responsável por uma doença perigosa, que tem sido veiculada na mídia (jornais; Globo - emissora de televisão aberta no Brasil). Ademais, ancora-se a sua transmissão através dos morcegos, o que pode ser justificado dado ao fato histórico, vivenciado em meados de 2003, de que esse mamífero fora indicado como um provável agente de disseminação da SARS (Watanabe et al., 2010).

Outro aspecto importante dessa classe relaciona-se ao papel simbólico e pivô da figura dos chefes de estado - presidentes - e seus posicionamentos neste momento de crise e disseminação do SARS-CoV-2. Contextualizando-se no cenário atual brasileiro, a evocação do termo presidente pode ou não se vincular aos posicionamentos do governo de Jair Bolsonaro, contrários às recomendações de quarentena feitas pelas instituições internacionais de saúde para contenção do vírus (WHO, 2020; International Federation of Red Cross and Red Crescent Societies, 2019). Em linhas gerais, o discurso desse governo parece justificar

a não-quarentena, por questões econômicas. Em pronunciamentos e entrevistas veiculados na mídia e hipermídia, seus apoiadores sugerem que um número aceitável de vidas podem ser perdidas, desde que a economia não pare. Os idosos, nesse contexto, na condição de grupo de maior risco, parecem também ser desconsiderados. Isso pode estar relacionado ao fato de que, em grande proporção, eles se encontram fora

8 do contexto laboral, sendo representados como incapazes e improdutivos (Araújo, Sá, \& Amaral, 2011) para 
a economia. Além disso, tais posicionamentos podem estar ancorados, ainda, na concepção de que apenas os mais fortes sobrevivem (seleção natural), na qual, sendo tal grupo considerado "fraco", pode morrer. Acerca dessas questões, sugere-se que estudos futuros busquem compreender de forma sistematizada o papel de chefes de estado em contextos pandêmicos, bem como os valores e as ideologias que ancoram posicionamentos da população em face do SARS-CoV-2.

Em oposição às classes 1 e 2, destacam-se as "Estratégias de enfrentamento do coronavírus e canais de informação", como características das evocações dos participantes residentes no Norte do Brasil. Para eles, a utilização de álcool, sabão e máscaras, bem como o isolamento, o evitamento de aglomerações e a quarentena mostram-se como fatores de prevenção e proteção frente à propagação do vírus. Cabe destacar, ainda, o papel da mídia - jornais impressos e televisivos, bem como revistas de circulação nacional - nessa apropriação (Simoneau \& Oliveira, 2015), uma vez que, de forma incisiva, tem feito a divulgação de notícias com dados de novos casos e pontos de disseminação, assim como a transmutação de estudos desenvolvidos em todas as partes do mundo para a população.

Por outro lado, a classe 3 - que aparece sozinha, constituindo um eixo em oposição às demais classes destaca-se por tangenciar questões relativas às implicações sociais, psicológicas e afetivas, não se restringindo aos aspectos biomédicos. Na referida classe, percebe-se a preocupação coletiva com a prevenção da COVID-19, para além de sua necessidade pessoal, o que foi objetivado pelo termo empatia, que coloca em perspectiva a existência de outras pessoas pelas quais se pode experimentar esse sentimento. Assim, o cuidado profilático é uma ação realizada para si, mas também para a proteção da coletividade. Nesse sentido, salienta-se que Hoffman (2003) considera a empatia como uma variável afetiva preditora de comportamentos pró-sociais.

Chama atenção também, nessa classe, a preocupação com as fakes news, fenômeno típico do contexto brasileiro, que gera um conjunto de desinformações sobre objetos sociais, como o novo coronavírus, contribuindo para a construção de representações sociais disfuncionais. Ainda, destacam-se as implicações psicológicas que estão associadas à nova dinâmica social imposta pelo SARS-CoV-2. Consoante a isso, a gravidade e as incertezas relativas a esse fenômeno social provocam emoções e estados psicológicos como o medo, o desespero e até mesmo o pavor. Sublinha-se que implicações psicológicas também já foram observadas em outros contextos sociais diante do novo coronavírus (Duan \& Zhu, 2020; Fiorillo \& Gorwooad, 2020).

As variáveis de ancoragens mais significativas nessa classe (sexo feminino, região Sul e renda per capita entre três e quatro salários mínimos), revelam marcadores importantes na forma de representar as implicações do SARS-CoV-2. O sexo feminino, representativo dessa classe, pode estar relacionado ao fato de serem as mulheres que culturalmente mais se preocupam com a prevenção de doenças e, portanto, que mais buscam os serviços de saúde, em comparação com os homens (Botton, Cúnico, \& Strey, 2017). A variável alusiva à região Sul do Brasil, considerada aquela que dispõe de maior poder aquisitivo, parece indicar que, quando as necessidades materiais de sobrevivência estão supostamente garantidas, é possível ampliar o olhar para as diligências de cunho coletivo e subjetivo (Qiu et al., 2020).

No que se refere ao estímulo "tratamento de pessoas com coronavírus", nota-se que o campo representacional desse objeto é constituído, majoritariamente, por elementos que remetem seja a indicações que podem ser seguidas para a remissão ou a amenização dos sintomas causados pela COVID-19, seja a estratégias de prevenção que devem ser adotadas frente à doença.

Observa-se que, na classe 1 (Estratégias de contenção e automedicação), a ancoragem social que mais contribuiu para a sua composição foi a renda (entre um e dois salários mínimos). Para os participantes com esse perfil socioeconômico, o tratamento de pessoas com coronavírus perpassa aspectos de prevenção e contenção mais relacionados a ações de cunho pessoal/individual. Nessa classe, destacam-se as palavras paracetamol, dipirona, remédios, antitérmico, nebulização e chá. Esses elementos podem indicar que a automedicação é uma prática comum entre as pessoas que apresentam os sintomas da COVID-19. Apesar 
de as medicações mencionadas pelos participantes serem apontadas como eficazes no combate aos sintomas leves causados pelo coronavírus, esse é um dado que chama a atenção, pois a automedicação pode acarretar efeitos negativos, sobretudo para aqueles que compõem o grupo de risco, como idosos e pessoas com doenças respiratórias que, em geral, fazem uso de outros medicamentos (fenômeno da polifarmácia), o que pode provocar efeitos colaterais devido à interação entre os fármacos (Secoli, Marquesini, Fabretti, Corona, \& Romano-Lieber, 2018).

Destaca-se, ainda, nessa classe o isolamento como uma forma de contenção da doença enfatizada pelos participantes. Aqui, faz-se importante compreender qual a definição de isolamento dos respondentes, tendo em vista que essa resposta de saúde pública ao surto da COVID-19 pode ter sérias implicações psicológicas, como o aumento da ansiedade e dos níveis de estresse (Duan \& Zhu, 2020; Xiang et al., 2020). Salienta-se que os órgãos e as autoridades de saúde enfatizam que não se trata de um isolamento social, mas sim de um distanciamento físico, estimulando o uso de canais de comunicação seguros para a manutenção do contato entre as pessoas, como forma de diminuir as consequências do distanciamento (Duan \& Zhu, 2020; Fiorillo \& Gorwooad, 2020).

A classe 2, intitulada "Implicações psicossociais e econômicas para o tratamento", foi representativa para aqueles respondentes com escolaridade até o ensino médio e com renda de até um salário mínimo. Para esses participantes, as estratégias de cuidado e prevenção frente ao novo coronavírus se ancoram em termos que indicam a necessidade de ações mais amplas, com ênfase no bem-estar coletivo. Destacam-se os elementos responsabilidade, cuidados, atenção, proteção, respeito, coletividade e prevenção. Nota-se que as particularidades das condições socioeconômicas desses respondentes encontram-se refletidas nessa classe, quando se observam elementos como pobres, risco, colapso e tristeza, enfatizando a situação de vulnerabilidade em que se encontram as pessoas de tal estatuto socioeconômico, tendo em vista que, em sua grande maioria, não têm acesso a itens de proteção e não podem parar suas atividades laborais, o que faz aumentar seus riscos de contaminação.

A esse respeito, um estudo realizado por Qiu et al. (2020), que buscou investigar o sofrimento psíquico na população geral da China durante a epidemia de COVID-19, demonstrou que os trabalhadores que precisavam se deslocar diariamente para o serviço experimentaram o mais alto nível de sofrimento psíquico, quando comparados àqueles que foram dispensados de suas atividades ou que estavam trabalhando em home office. Os altos níveis de estresse desses trabalhadores estavam relacionados à preocupação com a exposição ao vírus no transporte público para o trabalho, com a diminuição do tempo de trabalho e com a consequente diminuição de renda.

Esses dados corroboram as observações do presente estudo, demonstrando que, a depender do estatuto socioeconômico que ocupam, as pessoas estarão preocupadas não apenas com a prevenção e a contenção de uma determinada doença, nesse caso específico a COVID-19. Portanto, cabe às autoridades competentes levar em consideração a existência de uma realidade em que, ao lado da doença, as pessoas experimentam e incorporam várias incertezas cotidianas, associadas às suas condições precárias de vida, que afetam sua sobrevivência.

Tratando-se da classe 3 (Itens de proteção, suporte pessoal e assistência médico-hospitalar), que foi construída, essencialmente, pelas evocações dos participantes de alto poder aquisitivo para o contexto brasileiro (renda per capita superior a oito salários mínimos) e com ensino superior completo. Verifica-se que esses participantes enfatizam itens específicos de proteção, tais como máscara, álcool e vitamina, e, além disso, evocam elementos que apontam para a possibilidade de realização do cuidado em casa, como cama, que se diferencia de leito, que também aparece na classe, mas está relacionado ao cuidado médico-hospitalar. Tal como nas classes anteriores, as ancoragens sociais permitem observar que determinadas ações de cuidado estão restritas ao imaginário social daqueles que têm acesso a condições para realizar tratamentos específicos e obter determinados produtos. Nota-se que os elementos que compõem essa classe referem-se a termos 
específicos, como lavar, e a itens de cuidado também típicos, o que demonstra o conhecimento e a clareza desse grupo a respeito das medidas de prevenção e contenção do novo coronavírus, recomendadas pela comunidade científica (Adhikari et al., 2020).

Entretanto, vale salientar que, apesar de ter maior acesso a itens de proteção e ao cuidado médico-hospitalar, esse grupo não está isento das consequências psicológicas que a COVID-19 pode provocar. Estudos demonstram que pessoas com ensino superior tendem a sentir mais angústia em situações de emergências de saúde pública, provavelmente devido à alta autoconsciência que possuem de sua saúde (Qui et al., 2020).

Já a classe 4, constituinte única do eixo denominado a "Busca da cura da COVID-19: instituições e agentes responsáveis", revela o momento típico vivenciado pelos participantes no período da coleta dos dados deste estudo. Trata-se de um cenário em que o tratamento e a imunização dessa doença ainda são perpassados por incertezas e especulações (Mahase, 2020).

Essa classe é mais representativa dos participantes da região Sudeste que, vale ressaltar, foi onde primeiro foram testados e confirmados os casos de pessoas com a COVID-19 no Brasil (Macedo, Ornellas, \& Bomfim, 2020). O fato de, naquele momento, a doença fazer parte mais íntima da experiência dos participantes do Sudeste do que daqueles de outras regiões, desperta-Ihes a necessidade de controle de tal realidade, o que contribui diretamente para a formação de representações sociais objetivadas a partir dos seguintes aspectos: possíveis fontes (ciência e pesquisa), locais de tratamento (hospitais e SUS) e menção aos responsáveis pelo agenciamento da cura do novo coronavírus (governo, Deus, enfermeiros e médicos). Nesse sentido, as objetivações servem ao propósito de transformar uma realidade abstrata, como é o caso do tratamento da COVID-19, em algo cognoscível (Moscovici, 2017), ao menos do ponto de vista da esperança.

Em linhas gerais, indica-se que, a partir da TALP, os participantes que compuseram este estudo puderam compartilhar e nomear cognições e comportamentos forjados em meio social frente ao vírus SARS-CoV-2 e à doença que ele provoca, bem como às questões, ainda que especulativas, de seu tratamento. Nesse direcionamento, destaca-se o potencial dessa ferramenta de pesquisa para responder aos questionamentos propostos, permitindo capturar os elementos embrionários das RS do novo coronavírus e do tratamento da COVID-19.

Visando-se ampliar o espectro de compreensão das crenças, percepções, opiniões, ideias e práticas específicas das diferentes regiões do país acerca dos objetos sociais em estudo, sugere-se que pesquisas futuras visem ampliar, equalizar e homogeneizar as amostras de participantes, seja em relação ao sexo (uma vez que o presente estudo dispôs majoritariamente de mulheres), seja em relação à região do país (pois participaram, aqui, mais pessoas da região Nordeste).

Além dessas questões de organização metodológica, novos estudos devem buscar compreender o papel da simpatia ideológica e da empatia no contexto da COVID-19. Um possível modelo a ser testado seria a relação entre a simpatia ideológica (conservadores versus progressistas), a empatia e os comportamentos pró-sociais frente às pessoas contaminadas pelo SARS-CoV-2. Sugerem-se também estudos que busquem compreender a associação entre o poder aquisitivo e a percepção de vulnerabilidade ao novo coronavírus.

Salienta-se, ainda, que as análises deste estudo podem ser úteis para fundamentar estratégias interventivas por órgãos governamentais e não governamentais, bem como pela mídia, frente à COVID-19. Para fins de intervenção por tais agentes, aponta-se a necessidade de considerar as especificidades dos diferentes grupos sociais na forma como se apropriam de um saber reificado, típico do universo científico, como é o caso do novo coronavírus, e o transformam em um saber do senso comum. Isso porque o guia de leitura de uma mesma mensagem, por grupos distintos, é baseado nos elementos disponíveis em sua realidade e em sua experiência social. Logo, considerar tais aspectos pode garantir, ao menos, uma comunicação cognoscível, capaz de instrumentalizar a construção e a disseminação de representações e práticas sociais que confluam para a prevenção e a contenção do novo coronavírus no cenário brasileiro. 


\section{Contribuição}

Todos os autores participaram da concepção e delineamento do trabalho e participação na discussão dos resultados; redação do manuscrito e revisão crítica do seu conteúdo e aprovação da versão final do manuscrito.

\section{Referências}

Adhikari, S. P, Meng, S., Wu, Y. -J. Mao, Y. P., Ye, R. -X., Wang, Q. Z., ... Zhou, H. (2020). Epidemiology, causes, clinical manifestation and diagnosis, prevention and control of coronavirus disease (COVID-19) during thee earle outbreal period: a scoping review. Infectious Diseases of Poverty, 9(1), 1-12. http://dx.doi.org/10.1186/s40249-020-00646-x

Araújo, L., Sá, E. C. N., \& Amaral, E. B. (2011). Corpo e velhice: um estudo das representações sociais entre homens idosos. Psicologia: Ciência e Profissão, 31(3), 468-481. http://dx.doi.org/10.1590/S1414-98932011000300004

Botton, A., Cúnico, S. D., \& Strey, M. N. (2017). Diferenças de gênero no acesso aos serviços de saúde: problematizações necessárias. Revista Mudanças Psicologia da Saúde, 25(1), 67-72. http://dx.doi.org/10.15603/2176-1019/mud. v25n1p67-72

Brooks, S. K., Webster R. K., Smith L. E., Woodland L., Wessely, S., Greenberg, N., \& Rubin, G. J. (2020). The psychological impact of quarantine and how to reduce it: rapid review of the evidence. The Lancet, 395(10227), 912-920. http:// dx.doi.org/10.1016/S0140-6736(20)30460-8

Camargo, B. V., \& Justo A. M. (2018). Tutorial para uso do software de análise textual IRAMUTEQ. Florianópolis: Universidade Federal de Santa Catarina. Recuperado de http://iramuteq.org/documentation/fichiers/tutoriel-portugais-22-11-2018

Correia, M. I. D. T., Ramos, R. F., \& Bahten, L. C. V. (2020). Os cirurgiões e a pandemia do COVID-19. Revista Colégio Brasileiro de Cirurgiões, 47(1), 1-6. http://dx.doi.org/10.1590/0100-6991e-20202536

Coutinho, M. P. L., \& Do Bú, E. (2017). A técnica de associação livre de palavras sobre o prisma do Software tri-deuxmots (version 5.2). Revista Campo do Saber, 3(1), 219-242. Recuperado de http://periodicos.iesp.edu.br/index.php/ campodosaber/article/view/72/58

Do Bú, E. A., Alexandre, M. E. S., Bezerra, V. A. S., Sá-Serafin, R. C. N., \& Coutinho, M. P. L. (2020). Representações e ancoragens sociais do novo coronavírus e do tratamento da COVID-19 por brasileiros. Scielo Preprints. Versão 1. http://doi.org/10.1590/SciELOPreprints. 120

Doise, W. (2001). Direitos do homem e força das ideias. Lisboa: Livros Horizontes.

Duan, L., \& Zhu, G. (2020). Psychological interventions for people affected by the COVID-19 epidemic. The Lancet, 7(4), 300-302. http://dx.doi.org/10.1016/\$2215-0366(20)30073-0

Fiorillo, A., \& Gorwood, P. (2020). The consequences of the COVID-19 pandemic on mental health and implications for clinical practice. European Psychiatry, 63(1), 1-4. http://dx.doi.org/10.1192/j.eurpsy.2020.35

International Federation of Red Cross and Red Crescent Societies. (2019). Psychological first aid. Recuperado de https:// media.ifrc.org/ifrc/emergency/global-covid-19/

Hoffman, M. L. (2003). Empathy and moral development: implications for caring and justice. New York: University Press.

Huang, C., Wuang, Y., Xingwang, L., Ren, L., \& Zao, J. (2020). Clinical feature of patients infected with 2019 novel coronavirus in Wuhan, China. The Lancet, 395(10223), 497-506. http://dx.doi.org/10.1016/S0140- 6736(20)30183-5

Li, Q., Guan, X., Wu, P., Wang, X., Zhou, L., Tong, Y., ... Feng, Z. (2020). Early transmission dynamics in Wuhan, China, of novel coronavirus-infected pneumonia. New England Journal of Medicine, 382, 1199-1207. http://dx.doi.org/10.1056/ NEJMoa2001316

Macedo, Y. M., Ornellas, J. L., \& Bomfim, H. F. (2020). COVID - 19 no Brasil: o que se espera para população subalternizada? Revista Encantar: Educação, Cultura e Sociedade, 2, 1-10. http://dx.doi.org/10.5935/encantar.v2.0001

Mahase, E. (2020). COVID-19: what treatments are being investigated? BMJ, 368(1252), 1-2. http://dx.doi.org/10.1136/ bmj.m1252

Ministério da Saúde. (Brasil). (2016). Resolução n 510, de 7 de abril de 2016. Estabelece as diretrizes e normas regulamentadoras de pesquisas envolvendo seres humanos. Diário Oficial da União. Brasília: Autor. Recuperado de http://www.in.gov.br/materia/-/asset_publisher/Kujrw0TZC2Mb/content/id/22917581

Moscovici, S. (2017). A Psicanálise, sua imagem e seu público. Petrópolis: Vozes.

Oliveira, D. C. (2000). Social representations and public health care: subjectivity as a participant in the daily routine in health care. Revista de Ciências Humanas, 2, 47-65. http://dx.doi.org/10.5007/\%25x 
Qui, J., Shen, B., Zhao, M., Wang, Z., Xie, B., \& Xu, Y. (2020). A nationwide survey of psychological distress among Chinese people in the COVID-19 epidemic: implications and policy recommendations. General Psychiatry, 33, 1-3 http://dx.doi.org/10.1136/gpsych-2020-100213

Sá-Serafim, R. C. N. (2013). Corpo mastectomizado e representações sociais: rede de significações que conduzem a ação (Tese de doutorado não-publicada). Universidade Federal da Paraíba. Recuperado de https://repositorio.ufpb. br/jspui/handle/tede/6961

Secoli, S. R., Marquesini, E. A., Fabretti, S, C., Corona, L. P., \& Romano-Lieber, N. S. (2018). Tendência da prática de automedicação entre idosos brasileiros entre 2006 e 2010: estudo SABE. Revista Brasileira de Epidemiologia, 21(2), 1-14. http://dx.doi.org/10.1590/1980-549720180007.supl.2

Simoneau, A., \& de Oliveira, D. (2015). Representações sociais e meios de comunicação: produção do conhecimento científico em periódicos brasileiros. Psicologia e Saber Social, 3(2), 281-300. http://dx.doi.org/10.12957/psi.saber. soc.2014.14478

Velavan, T, P., \& Mayer, C. G. (2020). The COVID-19 epidemic. Tropical Medicine and International Health, 25(3), 278$280 \mathrm{http}: / / \mathrm{dx}$.doi.org/10.1111/tmi.13383

Villegas-Chiroque, M. (2020). Pandemia de COVID-19: pelea o huye. Revista Experiencia en Medicina del Hospital Regional Lambayeque, 6(1). http://dx.doi.org/10.37065/rem.v6i1.424

Watanabe, S., Masangkay, J. S., Nagata, N., Morikawa, S. Mizutani, T., Fukushi, S., ... Akashi, H. (2010). Bat Coronaviruses and Experimental Infection of Bats, the Philippines. Emerging Infectious Diseases, 16(8), 1217-1223. http://dx.doi. org/10.3201/eid1608.100208

World Human Organization. (2020). Coronavirus disease (COVID-19): situation dashboard. Geneve: Author. Retrieved from dehttps://experience.arcgis.com/experience/685d0ace521648f8a5beeeee1b9125cd

Xiang, Y., Yang, Y., Li, W., Zhang, L., Zhang, Q., \& Cheung, T. (2020). Timely mental health care for the 2019 novel coronavirus outbreak is urgently needed. The Lancet, 7(3), 227-229. http://dx.doi.org/10.1016/S2215-0366(20)30046-8

Xu, H., Zhong, L., Deng, J., Peng, J., Dan, H., Zeng, X., ... Chen, Q. (2020). High expression of ACE2 receptor of 2019-nCoV on the epithelial cells of oral mucosa. International Journal of Oral Science, 12(8). http://dx.doi.org/10.1038/s41368-0 20-0074-x

Recebido em: abril 13, 2020

Aprovado: abril 23, 2020 